\title{
Brief Report: The Effectiveness of Mirror Therapy on Upper Limb Function in Stroke Patients: A Single Case Experimental Design
}

\author{
Mehdi Rassafiani ${ }^{1,2 *}$ (D), Aisha Al-Fraij ${ }^{2}$ (D), Abrar Al-Mahri² (D), Fahad Manee ${ }^{1}$ (D) \\ 1. Occupational Therapy Department, Faculty of Allied Health, Kuwait University, Kuwait. \\ 2. Occupational Therapy Department, Physical Medicine and Rehabilitation Hospital, Ministry of Health, Kuwait.
}

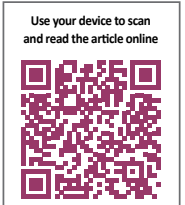

Citation: Rassafiani M, Al-Fraij A, Al-Mahri A, Manee F. The Effectiveness of Mirror Therapy on Upper Limb Function in Stroke Patients: A Single Case Experimental Design. Iranian Rehabilitation Journal. 2019; 17(3):285-292. http://dx.doi. org/10.32598/irj.17.3.285

http://dx.doi.org/10.32598/irj.17.3.285

Keywords:

Stroke, Upper Limb, Therapy

\section{ABSTRACT}

Objectives: To assess the effectiveness of Mirror Therapy (MT) on Upper Limb (UL) function of sub-acute stroke patients.

Methods: This study is a single case experimental design with two participants. Twenty minutes of MT were implemented four times a week over a period of four weeks. For baseline phase, repeated measurements were performed six times for one participant and four times for the other participant using Fugl-Meyer Motor Function Assessment (FMA), and it was applied twice a week during the intervention phase. Brunnstrom Recovery Stages (BRS) and Barthel Index (BI) were applied once before the baseline phase, as well as prior and after the intervention phase.

Results: Based on Percentage of Non-overlapping Data (PND) for FMA subtests, MT had questionable effectiveness on UL for both participants, and MT was unreliable intervention on wrist function. For the hand, of the second participant, MT had questionable effectiveness, whereas for the first participant it was unreliable intervention. For coordination, MT was fairly effective for the first participant and had questionable effectiveness for the second participant. BRS-Arm scores indicated improvements for the second participant from 3 (out of 6) to 4 BRS-Hand scores indicated improvements for the first participant from 5 (out of 6) to 6. No changes were shown in BI.

Discussion: This study revealed that MT had positive effects on some sections of UL motor function and coordination, whereas no change in wrist motor function was observed. No functional improvements in $\mathrm{BI}$ and slight difference in BRS were noticed. 


\section{Highlights}

- Mirror therapy is an easy to apply and low cost of intervention method.

- Proximal section of the upper limb responded to four weeks of Mirror therapy better than distal section.

- Mirror therapy improved upper limb coordination.

- Mirror therapy was not influential on functional skills of participants within four weeks of intervention.

\section{Plain Language Summary}

Mirror therapy is an easy to applied intervention for people with stroke. Therapists can apply this intervention with low cost. Only a mirror with an experienced therapist is required to apply this therapy in a clinic and at home. Mirror therapy applied for two people with stroke for four weeks. The results of the study showed that the movements in elbow was improved, but not for the wrist and finger. Also it helped to improved upper limb coordination. However, participants' daily activities were not improved. Further research is necessary to understand if this therapy can be more effective on daily activities if we increase the duration.

\section{Introduction}

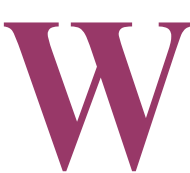

orldwide, stroke is a major contributor to chronic disabilities in adults causing Upper Limb (UL) dysfunctions that leading to functional deficits in activities of daily living [1]. Therefore, different rehabilitative interventions such as Mirror Therapy (MT) have been established to improve the functional performance of the UL [2].

MT can be implemented in three procedures, all of which involve a mirror in the midsagittal plane with the affected limb blocked by the mirror, and the patient facing the reflection of the non-affected limb movements [3]. MT is based on the concept of neuroplasticity of the brain. It is assumed that observing and executing an action might facilitate neural reorganization of the brain necessary for motor recovery $[4,5]$. The effectiveness of MT on patients with stroke was studied widely and showed mixed results. In 2012, results of a systematic review of literature showed that MT is moderately effective in improving motor performance of the hemiplegic UL, especially for sub-acute stroke patients [6].

Other two subsequent systematic reviews demonstrated similar results and showed improvement in UL function, activities of daily living and pain reduction. However, the certainty of evidence is not yet achieved requiring further research $[5,7]$. Following these reviews, another randomized controlled study with sham group was con- ducted recently and did not show any significant differences [8]. The previous studies showed that MT had positive effects on the UL function of stroke patients, especially if conducted within six months after the onset of stroke. However, there is no strong conclusions on which sections (distal or proximal) of UL function, it has more effectiveness. Therefore, this study aimed to assess the effectiveness of 20-minute sessions of MT on UL function (considering two sections) for sub-acute stroke patients. The results of this study will provide further evidence and improve our understanding about the effectiveness of MT on UL function and differences on its various sections.

\section{Methods}

\section{Methodology}

This study is a multiple baseline single case experimental design [9]. This method produces relatively high level of evidence when available resources are low and access to patients are limited.

\section{Participants}

Two sub-acute stroke patients from the neuro-inpatient department in the Physical Medicine and Rehabilitation (PMR) hospital that is a referral center for rehabilitation in Kuwait were recruited. They were included in the study if the onset of hemiplegia was within 6 months, had a Mini-Mental State Examination (MMSE) score of 24 or above, was between stages 3 and 4 of recovery 
for UL according to the Brunnstrom Recovery Stages (BRS), and had level 2 or lower in UL according to the Modified Ashworth Scale. Participants were excluded from the study if they had aphasia, and/ or accompanying disabling problems, such as vision or hearing impairments.

\section{Measurement tools}

Three measurement tools were utilized in this study. The first assessment was the Fugl-Meyer Motor Assessment (FMA) for measuring motor recovery after stroke. It contains 33 items for UL motor function that are scored based on a 3 -point ordinal scale $(0=$ cannot perform, $1=$ can partially perform, $2=$ can perform fully), and then scores are totaled to provide a maximum score of $66[10,11]$. FMA has an excellent construct validity, inter-rater reliability $(\mathrm{r}=0.97)$, and intra-rater reliability $(\mathrm{r}=0.99)$ [11].

The second assessment used was the BRS with high level of reliability (0.91-0.92) [12]. It is used to identify the stage of motor recovery in post-stroke patients based on the current level of spasticity and voluntary movement [13]. This test involves two items for UL (arm and hand), and is based on a 6-level Likert-type scale.

The third assessment was the Barthel Index (BI), which assesses the level of independency in activities of daily living and mobility. BI has high inter-rater reliability and responsiveness for stroke patients [14]. The BI yields five levels of function: 1. Very severe disability $(B I=20) ; 2$. Severe disability $(B I=25-45) ; 3$. Moderate disability $(\mathrm{BI}=50-70) ; 4$. Mild disability $(\mathrm{BI}=75$ 95); 5. No disability $(\mathrm{BI}=100)[14]$.

\section{Procedure}

Ethical approval was obtained from the Institutional Review Boards of Kuwait University (Approval number: 1863). All participants were informed about the whole process, purpose of the study, and their right to withdraw from the study at any time. At first, a pilot study with one participant with stroke was conducted and he received two weeks of MT. This pilot aimed to identify the time required to conduct the assessments and to ensure the client's safety and appropriate application of the intervention. Then, the main study was conducted with two participants that were randomly assigned into two different baseline periods in order to increase the internal validity [10].
The first participant was measured six times by FMA during three weeks of the baseline phase. This baseline was two weeks and four times of measurement for the second participant. In addition, at the beginning of the baseline phase for both participants, BI and BRS were administered once. After the baseline establishment, intervention started for four weeks, during this time FMA was applied twice a week and both BI and BRS were applied once at the beginning and once at the end of the intervention phase.

\section{Intervention protocol}

Twenty minutes of MT was implemented four times a week over a period of four weeks. The participants were positioned on a chair in front of a table, and the mirror was placed in the mid-sagittal plane, where the participant was only able to see the reflection of the unaffected arm. Then, participants were asked to mimic the movements of the unaffected arm with the affected arm if possible, or at least mentally imagining the movement. Every movement was executed in three sets; each set involved repeating the movement 20 times, and they had 30-second breaks after each set and between the tasks. Each week, a different set of exercises was performed started from proximal (pronation and supination) to distal [15].

\section{Analysis}

FMA scores were marked and recorded on a graph for visual analysis. Percentage of Non-overlapping Data (PND) was also calculated. PND is the percentage of data points during the intervention that are above the highest data point during the baseline. Results of the PND are interpreted as follows: the intervention is highly effective if PND exceeds (90\%); PND between (70\%) and $(90 \%)$ indicates a fairly effective intervention; For the results between $(50 \%)$ and $(70 \%)$, the intervention has questionable effectiveness; and results lower than (50\%) mean the intervention is unreliable [16].

\section{Resuts}

The first participant was a 53-year-old right-handed male, who had right Middle Cerebral Artery infarction four months prior to the study, resulting in left hemiplegia. He was admitted to hospital for rehabilitation six weeks prior to the study. The participant received physical therapy four times a week and occupational therapy twice a week. The participant missed two MT sessions in the third week of intervention and one assessment measure due to personal issues. He had experienced 
Participant 1
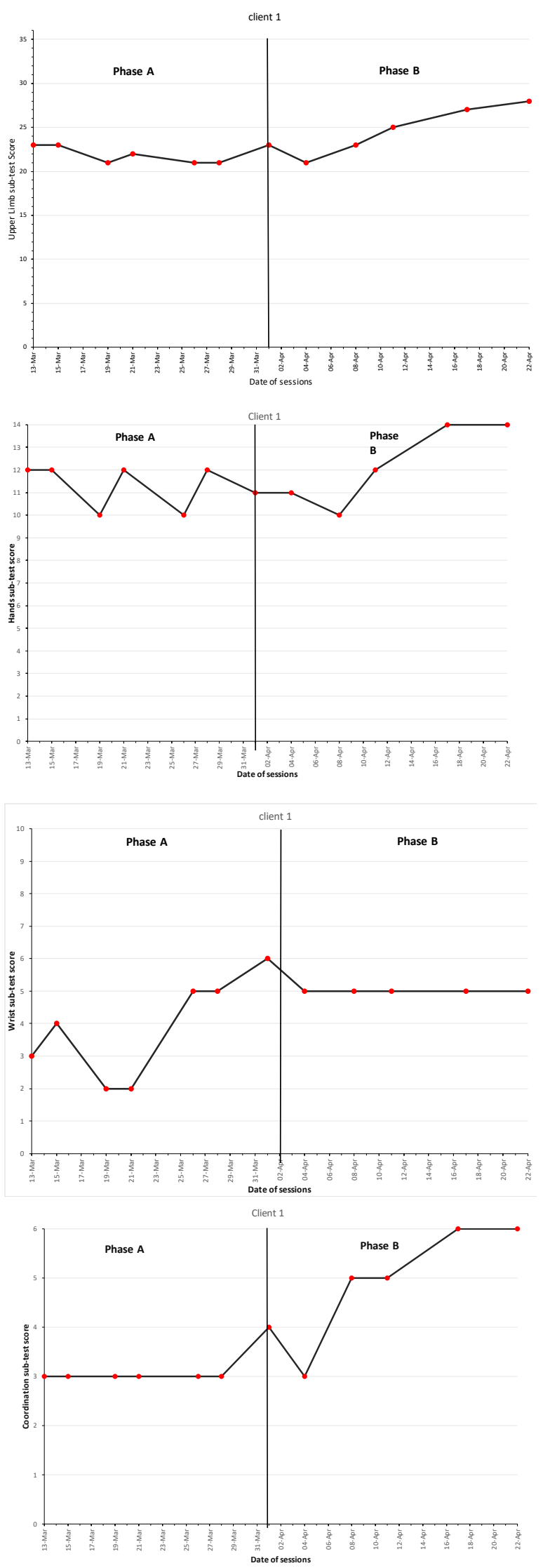

Participant 2
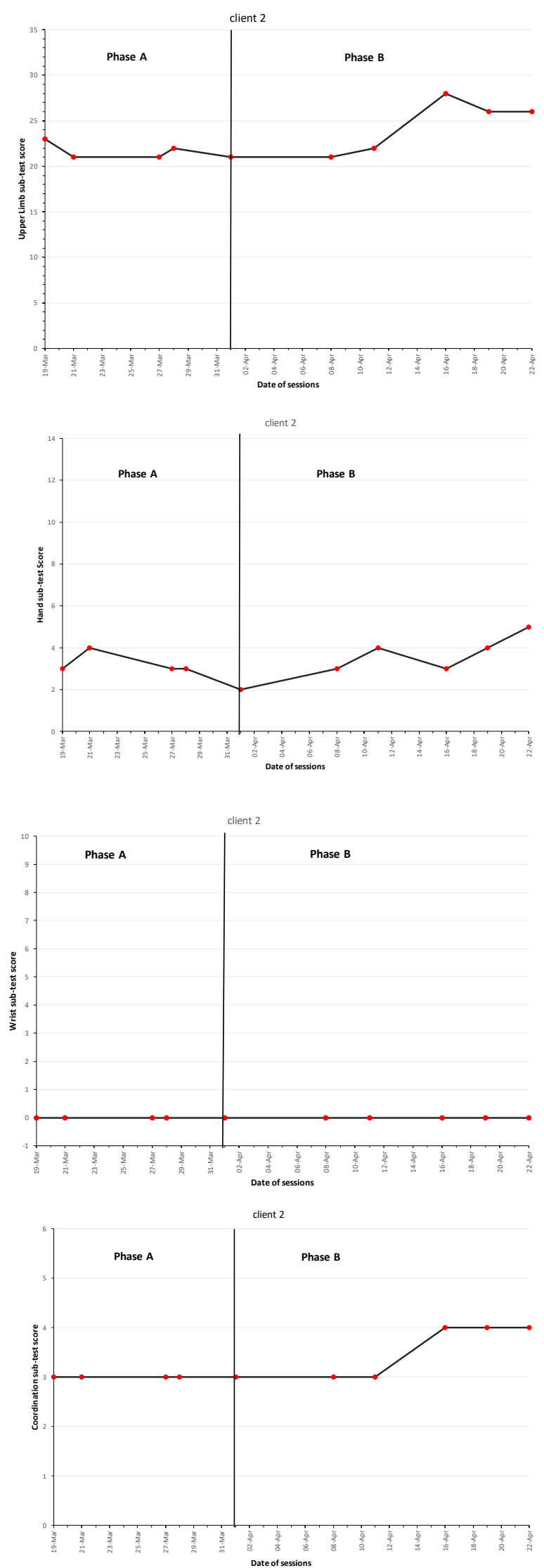

Figure 1. Fugl-Meyer Motor assessment subtest results for participants 1 and 2

Iranian Rehabilitation Journal 
Table 1. PND Results for FMA subtests

\begin{tabular}{ccccc}
\hline \multicolumn{5}{c}{ FMA Subtest } \\
\hline Participant Number & Upper Limb & Wrist & Hand & Coordination \\
\hline 1 & 57 & 14 & 43 & 86 \\
& (Questionable effectiveness) & (Unreliable) & (Unreliable) & (Fairly effective) \\
27 & 0 & 57 & 57 \\
& (Questionable effectiveness) & (Unreliable) & (Questionable effectiveness) & (Questionable effectiveness) \\
\hline
\end{tabular}

PND: Percentage of Non-overlapping Data; FMA: Fugel-Meyer Assessment

Iranian Rehabilitation Journal

Table 2. Results of BI and BRS

\begin{tabular}{ccccccc}
\hline & \multicolumn{5}{c}{ Intervention } \\
\cline { 2 - 7 } Outcome Measure & \multicolumn{5}{c}{ Participant 1 } & \multicolumn{3}{c}{ Participant 2 } \\
\cline { 2 - 7 } & Baseline & Pre-test & Post-test & Baseline & Pre-test & Post-test \\
\cline { 2 - 7 } BI & 50.100 & 50.100 & 50.100 & 60.100 & 80.100 & 80.100 \\
BRS-Arm & 4 & 4 & 4 & 3 & 3 & 4 \\
BRS-Hand & 5 & 5 & 6 & 3 & 3 & 3 \\
\hline
\end{tabular}

BI: Barthel Index; BRS: Brunnstrom Recovery Stages

Iranian Rehabilitation \ournal

left shoulder joint pain before admission to the study. Therefore, he received a subscapular nerve injection to minimize the pain. This was done in the third week and prior to the last three data points in the baseline phase. However, no change in UL function was recognized after the injection.

The second participant was a 21 year-old right-handed female, who had right-side hemorrhagic stroke due to arteriovenous malformation three months prior to the study, which resulted in left hemiplegia. She was admitted to the hospital two months prior to the study. She received four physical therapy sessions and four occupational therapy sessions per week. The participant missed two MT sessions and one assessment. Both participants received elbow and wrist supports in order to guide their movements only.

Figure 1 demonstrates the results of FMA for both participants during the two phases. The scores started to increase in the UL subtest for both participants between the 8th and 10th session. However, no progress was indicated in the wrist subtest. Increase in hand scores was limited in the last sessions for both participants. In regard to the coordination subtests, both participants showed positive change during the intervention.
Table 1 presents PND for FMA subtest scores for each participant. The results showed that MT varies between being as "an unreliable intervention" to a "fairly effective intervention" on the scores of FMA subtests.

Table 2 shows the results of BI and BRS scores for the participants. The results showed no change in BI during the intervention phase for both participants. BRS-Arm scores showed improvement in the second participant from 3 (out of 6 ) to 4 . However, no improvement was noticed for the first participant in the BRS-Arm scores. BRS-Hand scores increased for the first participant from 5 (out of 6) to 6 , but no improvement was noticed for the second participant in BRS-Hand scores.

\section{Discussion}

This study revealed that MT has positive effects on UL motor function. The proximal section of the UL showed higher improvement compared to the distal. This might be due to the sequence of motor recovery after stroke [17]. Such improvements in both sections of UL functions were congruent with the study undertaken by Lee et al. [18]. Additionally, both clients demonstrated noticeable improvement in coordination, which supports the results of Yun et al. [19]. In contrast, no change in wrist motor function was observed. Brunetti et al., in 2015 conducted a study to deter- 
mine the factors affecting the outcome of MT in sub-acute stroke patients with severe hemiparesis as responders and non-responders to MT.

This study concluded that the base level of voluntary movement is one main factor that affects potential motor recovery after MT [20]. As the participants in our study had very minimal voluntary movement in the wrist, we can interpret it as a potential reason for this result in their wrist motor function.

The results of this study demonstrated no functional improvement according to the Barthel Index. The intervention of this study was mainly used simple MT and this result is in-line with the previous studies within which their results showed that task-oriented MT was more beneficial in terms of self-care and motor recovery compered to simple MT [17, 21].

The results also showed a slight difference in BRS among the two participants. This might be due to the low sensitivity of the Brunnstrom scale as the participants had to achieve all the movements in the higher stage to move to that stage [12].

From the psychological aspect, both participants reported their satisfaction with MT as it gave them more sense of control in the affected UL, even though it was the reflection of the unaffected one. However, this is a report of the participants and further research to investigate the effects of MT on clients' psychological aspects is recommended. Due to limited resources, this study was conducted with two participants limited its generalizability to the population of the study. Therefore, it is suggested to repeat this study with more participants.

\section{Conclusion}

MT is non-invasive technique that can be easily implemented in a clinic or as a part of a home program. This study was able to provide more evidence and support for the effectiveness of 20-minute sessions of MT on UL function in sub-acute stroke patients. However, further well-designed experimental studies with follow-up are recommended on larger populations and over longer periods.

\section{Ethical Considerations}

\section{Compliance with ethical guidelines}

All participants were informed about study objectives and those agreeing to participate signed the informed consent forms. Ethical approval was obtained from the Institutional Review Boards of Kuwait University (Approval number:1863).

\section{Funding}

This This research was extracted from the research project (No: 2012.207) and was not supported financially.

\section{Authors' contributions}

Designing the study, analyzing the data, and preparing the draft of the article: Mehdi Rassafiani; Contributing in the research proposal and the first draft of the manuscript, collecting the data: Al-Fraij Aisha, Abrar Al-Mahri; Contributing in the research proposal and drafting the manuscript: Farhad Manee.

\section{Conflict of interest}

The authors declared no conflict of interest.

\section{Acknowledgments}

Much appreciation to Mihir Prasad for his contribution and to the participants for their cooperation in this study.

\section{References}

[1] Centers for Disease Control Prevention. Prevalence of stroke-United States, 2006-2010. Morbidity and Mortality Weekly Report. 2012; 61(20):379-82. [PMID]

[2] Hatem SM, Saussez G, della Faille M, Prist V, Zhang X, Dispa D, et al. Rehabilitation of motor function after stroke: a multiple systematic review focused on techniques to stimulate upper extremity recovery. Frontiers in Human Neuroscience. 2016; 10:442. [DOI:10.3389/fnhum.2016.00442] [PMID] [PMCID]

[3] Harmsen WJ, Bussmann JB, Selles RW, Hurkmans HL, Ribbers GM. A mirror therapy-based action observation protocol to improve motor learning after stroke. Neurorehabilitation and Neural Repair. 2015; 29(6):509-16. [DOI:10.1177/1545968314558598] [PMID]

[4] Selles RW, Michielsen ME, Bussmann JB, Stam HJ, Hurkmans HL, Heijnen I, et al. Effects of a mirror-induced visual illusion on a reaching task in stroke patients: Implications for mirror therapy training. Neurorehabil Neural Repair. 2014; 28(7):652-9. [DOI:10.1177/1545968314521005] [PMID]

[5] Yang Y, Zhao Q, Zhang Y, Wu Q, Jiang X, Cheng G, et al Effect of mirror therapy on recovery of stroke survivors: A systematic review and network meta-analysis. Neuroscience. 2018; 15(390):318-36. [DOI:10.1016/j.neuroscience.2018.06.044] [PMID] 
[6] Fong Mei Toh S, Fong KNK. Systematic review on the effectiveness of mirror therapy in training upper limb hemiparesis after stroke. Hong Kong Journal of Occupational Therapy. 2012; 22(2):84-95. [DOI:10.1016/j.hkjot.2012.12.009]

[7] Thieme H, Morkisch N, Mehrholz J, Pohl M, Behrens J, Borgetto B. et al. Mirror therapy for improving motor function after stroke. Cochrane Database of Systematic Reviews. 2018 2018(7):CD008449. [DOI:10.1002/14651858.CD008449.pub3] [PMID] [PMCID]

[8] Antoniotti P, Veronelli L, Caronni A, Monti A, Aristidou E, Montesano M, et al. No evidence of effectiveness of mirror therapy early after stroke: An assessor-blinded randomized controlled trial. Clinical Rehabilitation. 2019; 33(5):885-93. [DOI:10.1177/0269215518824737] [PMID]

[9] Rassafiani M, Sahaf R. Single case experimental design: An overview. International Journal of Therapy and Rehabilitation. 2010; 17(6):285-9. [DOI:10.12968/ijtr.2010.17.6.48151]

[10] Gladstone DJ, Danells CJ, Black SE. The Fugl-Meyer Assessment of motor recovery after stroke: A critical review of its measurement properties. Neurorehabilitation and Neural repair. 2002; 16(3):232-40. [DOI:10.1177/154596802401105171] [PMID]

[11] Sanford J, Moreland J, Swanson LR, Stratford PW, Gowland C. Reliability of the Fugl-Meyer assessment for testing motor performance in patients following stroke. Physical Therapy. 1993; 73(7):447-54. [DOI:10.1093/ptj/73.7.447] [PMID]

[12] Huang CY, Lin GH, Huang YJ, Song CY, Lee YC, How MJ et al. Improving the utility of the Brunnstrom recovery stages in patients with stroke: Validation and quantification. Medicine. 2016; 95(31):e4508. [DOI:10.1097/MD.0000000000004508] [PMID] [PMCID]

[13] Naghdi S, Ansari NN, Mansouri K, Hasson S. A neurophysiological and clinical study of Brunnstrom recovery stages in the upper limb following stroke. Brain Injury. 2010; 24(11):1372-8. [DOI:10.3109/02699052.2010.506860] [PMID]

[14] Duffy L, Gajree S, Langhorne P, Stott DJ, Quinn TJ. Reliability (inter-rater agreement) of the Barthel Index for assessment of stroke survivors: Systematic review and meta-analysis. Stroke. 2013; 44(2):462-8. [DOI:10.1161/ STROKEAHA.112.678615] [PMID]

[15] Lim KB, Lee HJ, Yoo J, Yun HJ, Hwang HJ. Efficacy of mirror therapy containing functional tasks in poststroke patients. Annals of Rehabilitation Medicine. 2016; 40(4):629-6. [DOI:10.5535/arm.2016.40.4.629] [PMID] [PMCID]

[16] Morgan DL, Morgan RK. Single-case research methods for the behavioral and health sciences. London: SAGE Publications; 2008. [DOI:10.4135/9781483329697]

[17] Park JY, Chang M, Kim KM, Kim HJ. The effect of mirror therapy on upper-extremity function and activities of daily living in stroke patients. Journal of Physical Therapy Science. 2015; 27(6):1681-3. [DOI:10.1589/jpts.27.1681] [PMID] [PMCID]

[18] Lee MM, Cho HY, Song CH. The mirror therapy program enhances upper-limb motor recovery and motor function in acute stroke patients. American Journal of Physical Medicine \& Rehabilitation. 2012; 91(8):689-700. [DOI:10.1097/ PHM.0b013e31824fa86d] [PMID]

[19] Yun GJ, Chun MH, Park JY, Kim BR. The synergic effects of mirror therapy and neuromuscular electrical stimulation for hand function in stroke patients. Annals of Rehabilitation
Medicine. 2011; 35(3):316-21. [DOI:10.5535/arm.2011.35.3.316] [PMID] [PMCID]

[20] Brunetti M, Morkisch N, Fritzsch C, Mehnert J, Steinbrink J, Niedeggen $\mathrm{M}$, et al. Potential determinants of efficacy of mirror therapy in stroke patients: A pilot study. Restorative Neurology and Neuroscience. 2015; 33(4):421-34. [DOI:10.3233/ RNN-140421] [PMID] [PMCID]

[21] Paik YR, Kim SK, Lee JS, Jeon BJ. Simple and task-oriented mirror therapy for upper extremity function in stroke patients: A pilot study. Hong Kong Journal of Occupational Therapy. 2014; 24(1):6-12. [DOI:10.1016/j.hkjot.2014.01.002] 
This Page Intentionally Left Blank 